\title{
Report of the IAU WGAS Sub-group on Standard Procedures
}

\author{
T. Fukushima \\ National Astronomical Observatory; 2-21-1, Ohsawa, Mitaka, Tokyo 181; JAPAN; (Internet) \\ toshio@spacetime.mtk.nao.ac.jp
}

\section{Introduction}

The given mission of the subgroup on Standard Procedures (SGSP) of IAUWG on Astronomical Standards (IAU/WGAS) is to prepare report "on standard procedures needed in fundamental astronomy, which a) should have a maximum degree of compatibility with the IERS Standards, b) should include the implementations of procedures in the form of tested software and/or test cases, c) should be available not only in written form, but also in machine-readable form" as described in the third item of Recommendation VIII of the IAU Resolution A4 (1991). After some general discussions, in 1992 we issued a questionnaire on the image and mechanism on the supposed set of standard procedures (IAU/WGAS Circulrar 51.2.1) and sent it to over 200 scientists in the world. Within a few months, 16 answers were sent back. The detailed answers and the questionnaire was distributed (IAU/WGAS Circular 54.2.1). As a simulation of supposed mechanism, in 1993 we did a test campaign to collect computational procedure(s) to compute the present IAU precession formula (Lieske et al., 1979) in FORTRAN (IAU/WGAS Circular 59.2.1). Within a few months, 5 submissions from 5 countires (Japan, China, USA, Russia, and UK) reached. The analysis of submitted routines was circulated (IAU/WGAS Circular 65.2.1). Based on these responses, we concluded that the establishment of a mechanism to provide such procedures to the community of astronomy is quite useful. To realize this, we will recommend some action items to the IAU as is described in the next Section. Its main purpose is to establish a mechanism by the combination of a comiittee of board and a center, which are to operate within a year after the recommendations are adopted by the IAU. Section 3 will describe some guidelines for the committee and center to perform the tasks to create and maintain standard procedures. Of course, these are to be consulted if necessary and are not to restrict or regulate the activity of the committee and the center.

\section{Recommendations}

It is a significant task for the IAU to create and maintain a set of basic software which are computing commonly used formulas and standard models in the fundamental astronomy in order to enhance the interchangablity of observational data whether processed or not and theoretical ideas. This set should also cover the lists of constants and some astronomical quantities. Such set shall be called the IAU Standards of Fundamental Astronomy (SOFA). Therefore, we recommend the IAU to realize followings;

1. The IAU WGAS shall be in responsible to determine the scope and items of the SOFA.

Notes: a) Unnecessary overlap with the scope of other WGs and Commissions should be avoided.

b) For the moment, it is desirable to limit the covered items will be minimally sufficient.

2. A center at a suitable institution under a worldwide reviewing board should be in charge of the maintainance of this standards.

Notes: a) This center should take over the tasks recommended by the IAU WGAS Sub-group on Electronical Distribution. 
b) The center and reviewing board shall be ranked as formal functions of the IAU WGAS. The director of the center shall be a member of the IAU WGAS and a member of the reviewing board as well. The chair of reviewing board shall be a member of the IAU WGAS.

c) The IAU WGAS shall select and nominate the center and reviewing board within a year after the adoption of this Recommendation by the IAU. The nominated center and reviewing board shall start the mission within a year after the nomination.

3. The center and the IAU WGAS should do their best efforts to make the SOFA be maximally conforming to the IERS Standards and the resolutions of IAG Special Commission of Fuadamental Constants (SCFC).

Note: To assure this, the IAU WGAS shall welcome a representative from the IERS and another from the IAG SCFC. The IAU WGAS should seak for a possibility to operate the SOFA and other related standards under a joint service with the IERS and the IAG, and should report on the possibility to the next General Assembly of the IAU.

4. The SOFA shall be availlable in a machine-readable form.

Note: The center should set up an anonymous ftp service or similar passive system so that the SOFA is accessible electronically. It also should provide a service to send a part of the SOFA back in a floppy disk or some suitable electronical media on request from users.

5. The SOFA, its part or as a whole, shall not be used for profittable purposes unless the WGAS approves. The SOFA can be modified by users for their own use.

Note: It is desirable for the authors of SOFA to agree with this policy.

\section{Guidelines}

In the followings, we will present some guidelines for the center and the reviewing board specified in Recommendation 3 in the preceding section. These are based on the responses to the questionnaires (IAU/WGAS Circular 54.2.1) as well as the experiences on the test campaign which is partly viewed in the analysis report (IAU/WGAS Circular 59.2.1).

1. Guideline of Creation/Maintainance of Standard Procedures

1.1 The SOFA shall be self-consistent.

Note: Any program unit of the SOFA shall be designed to contain necessary materials in it, say data tables and/or sub-programs called by the program unit itself. To avoid unneccesary duplication of program codes, the calling of other program units of the SOFA is recommended as much as possible.

1.2 The formal computer languages of the SOFA are Fortran 77 and $\mathrm{C}$.

Notes: a) The selection of languages is provisional. According to the requirements, the policy will be re-examined.

b) This does not mean that the center and/or the reviewing board should present all procedures in each of formal languages at the same time.

c) The procedures aiming the same purpose but written in different computer languages are treated as different items of standard procedures. 
d) A rewriting of existing standard procedure of the SOFA into another computer language is regarded as a creation of new item.

1.3 Each standard procedure shall contain

1) a document to specify the purpose of procedure,

2) a single interface,

3) one or multiple set(s) of program units realizing the computational procedure,

4) one or multiple main program(s) testing the computation procedrue through this single interface,

5) one or multiple set(s) of $I / O$ examples obtained by the above test program,

6) document(s) explaining the minimum information on the above items 2)-5).

Notes: a) This is a compromise of the One-Realization-One-Item (OROI) policy and the MultipleRealizations-One-Item (MROI) policy. Namely, we admit multiple sets of realizations for Items 3)-5). But we unify the first two Items.

b) The interface means the combination of

- the name and characteristics of program units to be called,

- the name, characteristics, definition, and order of of arguments to be passed from/to the calling units.

c) The specification document should be prepared by the reviewing board before inviting Items 3)-6) from the public.

d) The interface should be specified by the reviewing board. The decision may be before and/or after inviting the submission.

e) The unification of interface can be realized by the technique of wrapping. The wrapping of submitted computational procedures is the task of the reviewing board.

f) The minimum information means the information without which the users can not use the program such as the specification of data files necessary.

g) The documents for Items 3)-5) should be written by the authors. In the case the reviewing board create/obtain Items 3)-5) by itself, it should prepare them.

h) The documents for the interface should be written by the reviewing board.

i) The documents shall be presented as comments in program units, or in separate text file(s).

2. Guideline of Collection of Standard Procedures

2.1 The SOFA consists of a group of relatively small standard procedures.

Note: The SOFA does not cover a large software package for the moment.

2.2 Each standard procedure corresponds to an item of computational procedure in the fundamental astronomy. The selection of item and its specification shall be done by the reviewing board.

Note: The items for the first couple of years may be

- 1976 IAU Precession (This is almost finished, but...)

- 1980 IAU Nutation

- UTC-TAI transformation

- TCB-TDB-TT transformation 
- GST computation

- Handling planetary ephemerides

Namely, the routines to provide the SSB (and other types of) position and velocity of solar system objects (the Sun, planets, natural satellites, asteroids, ....).

- Referencing star catalogues

Namely, the routines to provide the J2000.0 (or B1950.0 in some cases?) direction vector and its time derivative of stars from the star catalogues availlable.

- Catalog transformation from B1950.0 to J2000.0.

- Computation of the SSB position of an observer on the Earth

- Computation of the apparent/astrometric places of stars from an observer on the Earth

2.3 The center and the reviewing board prepare standard procedures by inviting submittance from the public. They should express the appreciation to the contributors through the newsletter.

The process of preparation will be;

1) The board selects an item and a principal reviewer who is in charge of preparing the specification and reviewing.

2) The principal reviewer prepares the specification document. The interface can be specified if he/she decides.

3) The center announces the invitation by showing the specification document and the deadline ( 2 months maybe a good choice). The address of submission is the center.

4) The center send the submitted material to the principal reviewer after a screening; check of the format of submission (, say the language, the machine readability, forms of attached documents).

5) The principal reviewer reviews the submissions;

- check of submitted programs at multiple computers and compilers,

- modification of announced official interface if necessary,

- preparation of the official test program based on the interface by selecting or combining the submitted test programs or by creating by himself,

- wrapping of the submitted computational programs to the official interface if necessary,

- comparison of the outputs by the official test programs at multiple computers,

- writing a review report,

- arrangement of a candidate member of SOFA as a package of a) specification, b) official interface, c) official test program, d) wrapped computational programs (which are recommendable), and e) official input/output examples.

6) The reviewing board examines the report to certify the proposed candidate

7) The center installs the new member into the SOFA and announce it.

Note: A sample timetable of inviting computational procedures is shown in Table I. This assumes 5 months for a total period per item.

2.4 The pace of collection is four items every year for the moment.

Note: This means that the collection should be done in a pipeline.

3. Guideline of Provision of Standard Procedures

3.1 The center shall open and maintain the anonymous ftp service providing

1) The SOFA, 
TABLE I

Model Schedule of Colletion of SOFA Routine

\begin{tabular}{lll}
\hline Weeks & Center & Reviewing Board \\
\hline $1-2$ & & $\begin{array}{l}\text { Selection of an item and a principal reviewer } \\
\text { Preparation of specification document by the principal reviewer }\end{array}$ \\
$3-4$ & & \\
5 & Announcement of invitation & \\
$5-12$ & Receiving and screening & Review by the principal reviewer \\
$13-16$ & & Examining by the board \\
$17-18$ & & \\
18 & Installation & \\
20 & Announcement of completion & \\
\hline
\end{tabular}

2) Non-confidential part of the reviewing reports,

3) Backnumbers of the newsletter (See 3.2),

Notes: a) The center can add some useful files to its anonymous ftp service if the reviewing board admits.

b) Other anonymous ftp sites can mirror the contents if the reviewing board admits.

3.2 The center shall issue periodically the newsletter containing

1) Announcements on inviting programs,

2) Status of activity of the center and the reviewing board,

3) Introduction of newly adopted standard procedure(s),

and send them to those who subscribe it.

Notes: a) The center can add any useful information to the newsletter.

b) The frequency of issuing is more than once per three months.

c) The subscribing should be free.

d) The E-mail is the most preferrable media of sending the newsletter. However, the center should send them via ordinary mail to those who have no good accesibility to the E-mail.

3.3 The center shall send back a part or the whole of the items in 3.1 in a machine-readable media upon request.

Note: The center can limit the media. For the moment, a 5.25 inch and/or a 3.5 inch MS-DOS formatted floppy is recommended as the minimum.

3.4 The center can charge a reasonable fee for this copy service.

Note: In case of charging, the amount of fee should be admitted by the WGAS.

3.5 To lessen the labor of the center as well as the total cost and time for the requesting people, the center can afford other institutions to take over this service for a limited area if the reviewing board admit. 